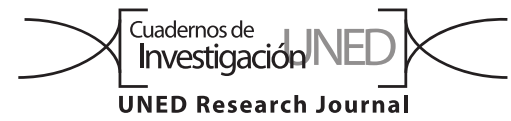

\title{
El árbol Myroxylon peruiferum (Fabaceae), nuevo registro en la flora de Costa Rica
}

\author{
Diego Aguilar-Sandí \\ 1. Universidad de Costa Rica, Escuela de Biología, 11501 San Pedro, Montes de Oca, Costa Rica; \\ dd.10306@gmail.com, (iD https://orcid.org/0000-0003-3909-5425 \\ Recibido 14-VI-2019 • Corregido 18-VIII-2019 • Aceptado 11-IX-2019
}

DOI: https://doi.org/10.22458/urj.v11i3.2722

\begin{abstract}
The tree Myroxylon peruiferum (Fabaceae), a new record for the Costa Rican flora". Introduction: The genus Myroxylon (Fabaceae) includes two Neotropical species, M. balsamum and $M$. peruiferum, both present from southern Mexico to South America. Despite this wide distribution, only the presence of $M$. balsamum was recognized in Costa Rica. Objectives: to confirm the presence of $M$. peruiferum in Costa Rica and to present the morphological characteristics for its differentiation from M. balsamum. Methods: In April 2019, three sites southwest of the Central Valley were explored. Botanical material was collected from trees of the Myroxylon genus and identified at the species level. Additionally, the collections of herbaria CR, JVR and USJ were reviewed. Results: $M$. peruiferum trees were found in the three explored sites, differentiable from $M$. balsamum in the morphology of the leaflets, flowers, mature fruits and seeds. $41 \%$ of the herbarium specimens identified as $M$. balsamum actually correspond to $M$. peruiferum. Conclusion: the absence of $M$. peruiferum in the records of the Costa Rican flora was due to the incorrect identification of the collected material and the ignorance that the species can be identified by vegetative characters.
\end{abstract}

Key words: Balsam, chirraca, native species, Acosta, El Rodeo, Salitral.
RESUMEN: Introducción: El género Myroxylon (Fabaceae) incluye dos especies, M. balsamum y M. peruiferum, presentes desde el sur de México hasta Suramérica. Sin embargo, en Costa Rica solo se reconocía la presencia de $M$. balsamum. Objetivos: confirmar la presencia de $M$. peruiferum en Costa Rica y presentar las características morfológicas para su diferenciación de M. balsamum. Métodos: En abril de 2019 se exploraron tres sitios al suroeste del Valle Central. Se recolectó material botánico de árboles del género Myroxylon y se identificó a nivel de especie. Adicionalmente, se revisaron las colecciones de los herbarios $C R$, JVR y USJ. Resultados: En los sitios explorados se encontraron árboles de $M$. peruiferum, diferenciables de $M$. balsamum en la morfología de los foliolos, las flores, los frutos maduros y las semillas. El $41 \%$ de los ejemplares de herbario identificados como M. balsamum corresponden a M. peruiferum. Conclusión: la ausencia de $M$. peruiferum en los registros de la flora costarricense se debió a la incorrecta identificación del material recolectado y al desconocimiento de que las especies pueden identificarse mediante características vegetativas.

Palabras clave: Bálsamo, chirraca, especie nativa, Acosta, El Rodeo, Salitral.
El género Myroxylon L. f. (Fabaceae, Faboideae) incluye dos especies, M. balsamum (L.) Harms y M. peruiferum L. f., nativas del Neotrópico y presentes desde el sur de México hasta el norte de Argentina y Brasil (Sartori et al., 2015). Ambas son árboles que alcanzan de 30 a 50m de altura, presentan peridermis grisácea, lenticelada, estípulas deciduas, hojas alternas, imparipinnadas, foliolos alternos, con puntos y rayas translúcidos, inflorescencias racemosas, flores zigomorfas, frutos samaroides y semillas reniformes (Zamora, 2010; Sartori et al., 2015). La madera es aromática, de muy pesada a extremadamente pesada $\left(>0,72 \mathrm{~g} / \mathrm{cm}^{3}\right)$ y resistente a la pudrición; usada en ebanistería y en construcción (Jiménez, Rojas, Rojas,
\& Rodríguez, 2011; Limongi, Guiracocha, \& Nieto, 2012). Los árboles de $M$. balsamum son la fuente del bálsamo del Perú, resina extraída del tronco y utilizada en la fabricación de aromatizantes, barnices, cosméticos, jarabes, inciensos, pomadas, entre otros (Martínez-Rivera, Blanco, Colorado, Guerra, \& Antonio, 2015).

En Costa Rica, M. balsamum se encuentra en zonas bajas (0-850m) y húmedas de la vertiente pacífica (Zamora, 2010); se considera una especie en peligro de extinción y su aprovechamiento está vedado (Decreto Ejecutivo No 25700-MINAE, 1997; Jiménez, 1998). En el caso de M. peruiferum, a pesar de su amplia distribución en el Neotrópico, su presencia en Costa Rica no había sido 
confirmada. Sartori et al. (2015) incluyeron en el tratamiento de M. peruiferum el ejemplar Cascante 1324 (CR), recolectado en El Rodeo, cantón de Mora, San José; sin embargo, este hecho fue puesto en duda por tratarse de una muestra estéril y por considerarse que no era posible diferenciar vegetativamente entre ambas especies (Grayum, Hammel, \& Zamora, 2016).

Este trabajo tiene como objetivos confirmar la presencia de M. peruiferum en Costa Rica y presentar las características morfológicas para su diferenciación de $M$. balsamum. Adicionalmente, se brinda información sobre su distribución y nombre vernáculo.

\section{MATERIALES Y MÉTODOS}

Sitios de estudio: En abril de 2019 se exploraron tres sitios en las estribaciones montañosas al suroeste del Valle Central, provincia de San José, Costa Rica: Alto Raicero-Chirracal (Salitral, Santa Ana, 0954'22.6" N, 84¹0'11.0" W, 1220-1299m), El Rodeo (Ciudad Colón, Mora, 0954'59.5" N, 84¹5'54.4" W, 900m) y San IgnacioChirraca (San Ignacio, Acosta, 0948'41.4" N, 84¹0'38.0" W, 980-1097m). Según el sistema de zonas de vida de Holdridge, estos sitios corresponden a bosque húmedo premontano y bosque muy húmedo premontano (Hartshorn, 1991; Bolaños, Watson \& Tosi, 2005); presentan estación seca de diciembre a abril y lluviosa de mayo a noviembre (Heuveldop \& Espinoza, 1983; Meza \& Bonilla, 1990; Cascante-Marín \& Estrada-Chavarría, 2012).

Se recolectó material botánico de al menos un árbol del género Myroxylon en cada sitio. Los testigos se depositaron en el herbario USJ (Luis A. Fournier Origgi, Escuela de Biología, Universidad de Costa Rica). Las coordenadas geográficas y la altitud se determinaron mediante una terminal de posicionamiento global GPSmap 60CSx marca Garmin.

Identificación del material: Las muestras recolectadas se identificaron tomando en cuenta el tratamiento de Sartori y colaboradores (2015) y un ejemplar con flores de $M$. peruiferum recolectado en febrero de 2019 de un árbol cultivado en el campus de la Universidad de Costa Rica, Montes de Oca, San José (Aguilar-Sandí 6, USJ).

Revisión de herbarios: Se revisaron las colecciones del género Myroxylon de los herbarios CR (Herbario Nacional, Museo Nacional de Costa Rica), JVR (Juvenal Valerio Rodríguez, Escuela de Ciencias Ambientales, Universidad Nacional de Costa Rica) y USJ (Luis A. Fournier Origgi, Escuela de Biología, Universidad de Costa Rica).

Ética, conflicto de intereses y declaración de financiamiento: El autor declara haber cumplido con todos los

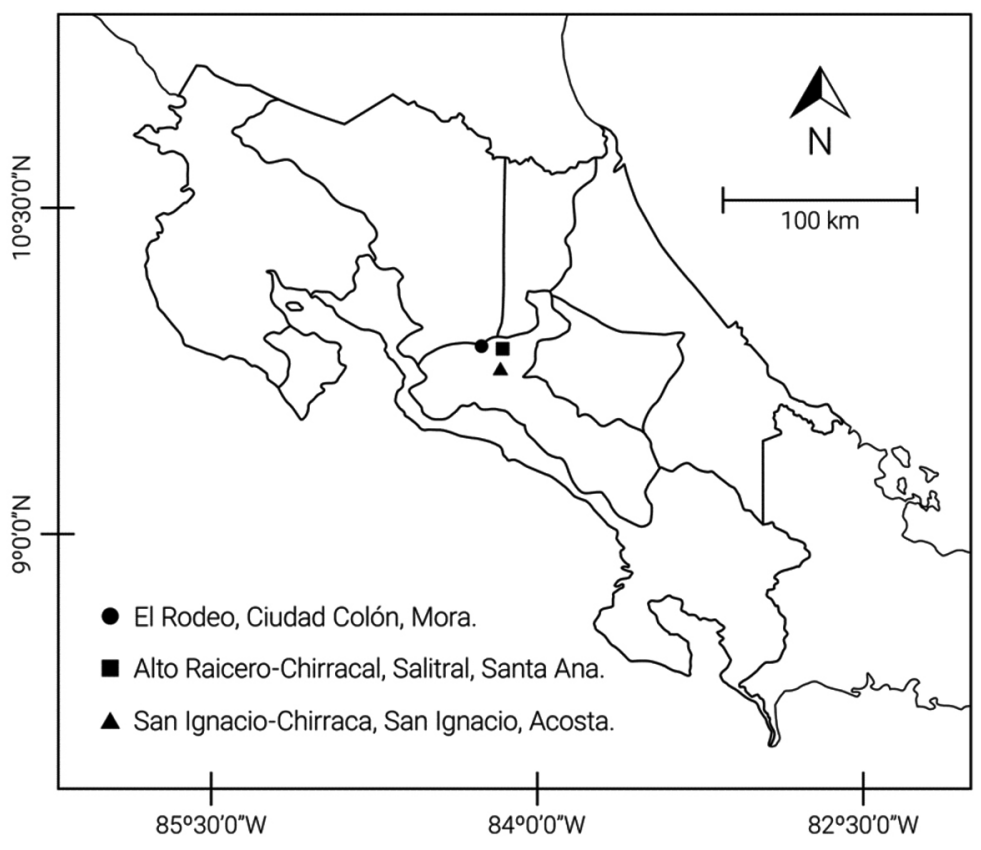

Fig. 1. Ubicación de árboles de Myroxylon peruiferum en las estribaciones montañosas al suroeste del Valle Central, San José, Costa Rica. 
requisitos éticos y legales pertinentes, tanto durante el estudio como en el manuscrito; que no hay conflictos de interés de ningún tipo, y que todas las fuentes financieras se detallan plena y claramente en la sección de agradecimientos. Asimismo, está de acuerdo con la versión editada final del documento. El respectivo documento legal firmado se encuentra en los archivos de la revista.

\section{RESULTADOS}

Recolectas e identificación: Se encontraron árboles de M. peruiferum en los tres sitios explorados (Fig. 1). En Alto Raicero-Chirracal los árboles estaban confinados a laderas montañosas rodeadas de fincas y cafetales. En El Rodeo los árboles estaban en un fragmento boscoso cercado por potreros. En San Ignacio-Chirraca se observaron árboles dispersos e inmersos en el paisaje suburbano. Se observó regeneración natural en los tres sitios.

Myroxylon peruiferum L. f., Supplementum Plantarum 233. 1781.

Tipo: America meridionalis, s.d., Mutis s.n. (holotipo: P 00679272)

Myroxylon peruiferum se diferencia de $M$. balsamum por tener foliolos más pequeños $[2,9-4,5(-10,5) \times(1,0-)$ $1,7-2,0(-3,3) \mathrm{cm}$ vs. 5,5-10,5 ×2,0-5,0cm], ápices retusos a acuminados (vs. acuminados a largamente acuminados) (Fig. 2), puntos y rayas translúcidos evidentes (vs. poco evidentes), flores con cáliz no lobulado (vs. cáliz lobulado) y estandarte ampliamente ovado (vs. estrechamente ovado) (Fig. 3), frutos maduros con cámara seminífera elíptico-aplanada (vs. subglobosa) y semillas de testa rugosa (vs. lisa) (Fig. 4) (Sartori et al., 2015). Ambas especies producen frutos con una o dos semillas.

Distribución: Estribaciones montañosas de la Cordillera de Talamanca al suroeste del Valle Central, entre 900 y $1230 \mathrm{~m}$ de altitud.

Nombre vernáculo: Los habitantes de San Ignacio de Acosta y de Salitral de Santa Ana conocen a M. peruiferum como chirraca, siendo el nombre común de esta especie el que da nombre a dos de los pueblos visitados: Chirraca de Acosta y Chirracal de Santa Ana (J. Madrigal \& I. Badilla, com. pers., 2019).

Revisión de herbarios: Todos los ejemplares del género Myroxylon depositados en los herbarios consultados, y recolectados antes de 2019, fueron identificados como M. balsamum. Sin embargo, el 41\% corresponden a $M$. peruiferum. Los ejemplares que sí corresponden a $M$. balsamum provienen, principalmente, de la zona sur del país (vertiente pacífica) entre 0 y 100m de altitud.

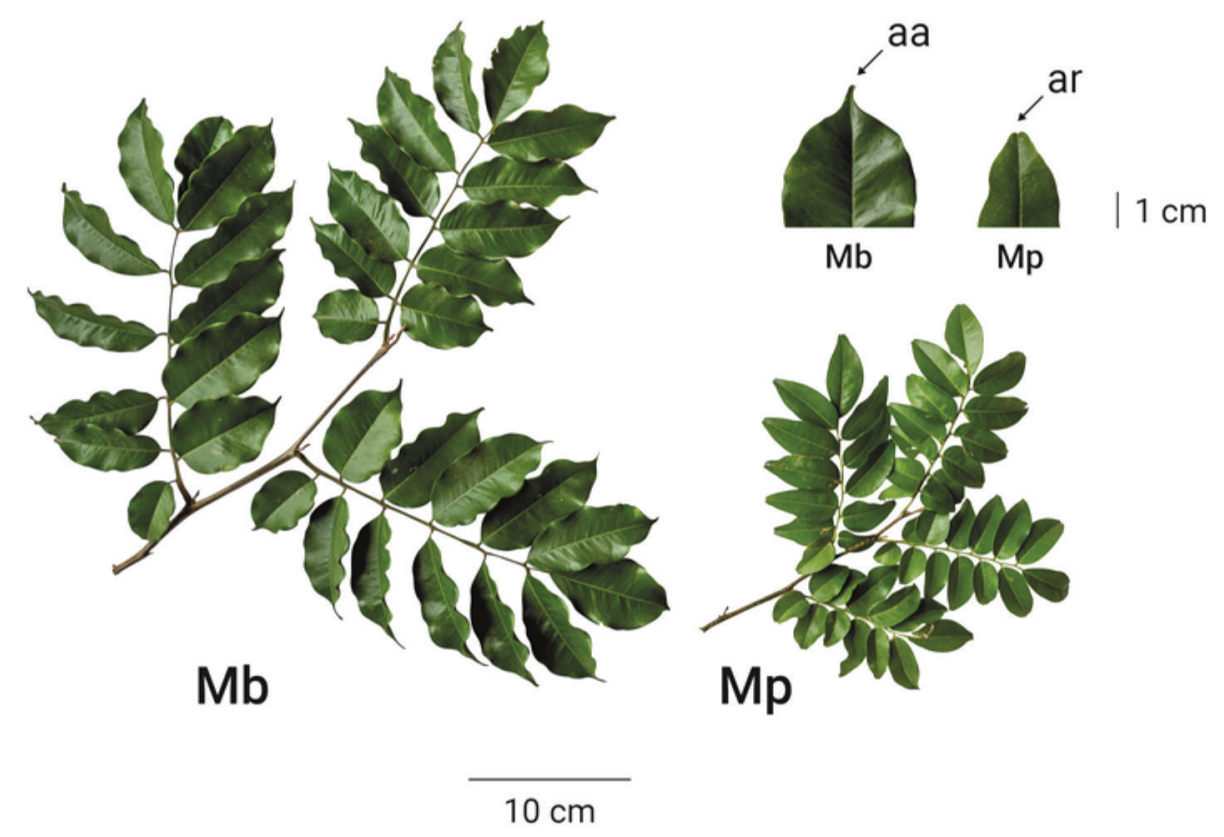

Fig. 2. Características morfológicas vegetativas para identificar y diferenciar Myroxylon peruiferum (Mp) de M. balsamum (Mb). Hojas y detalle de los foliolos (aa= ápice acuminado; ar= ápice retuso). 

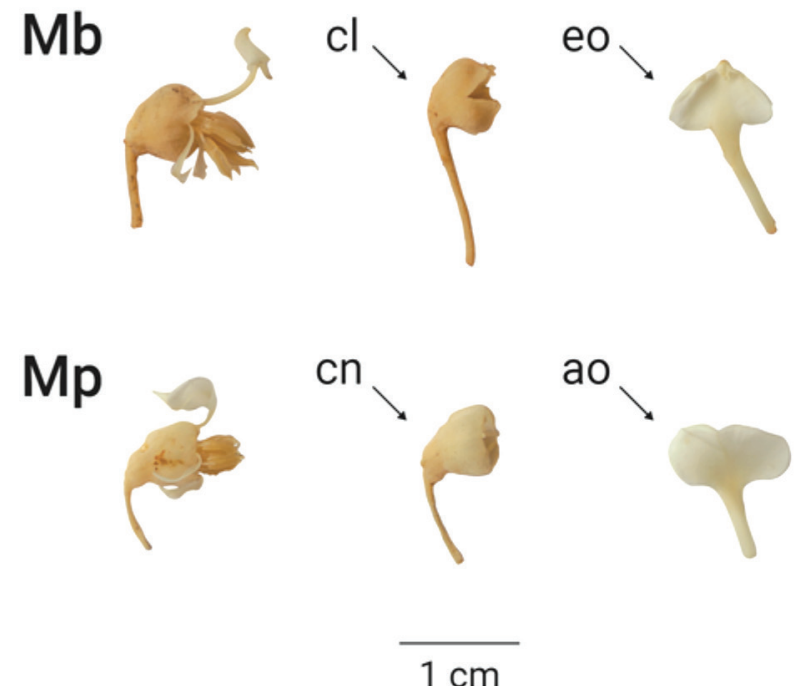

$1 \mathrm{~cm}$

Fig. 3. Características morfológicas florales para identificar y diferenciar Myroxylon peruiferum (Mp) de M. balsamum (Mb). Flores en etanol al $80 \%$ ( $c$ = cáliz lobulado; $c n=$ cáliz no lobulado; eo= estandarte estrechamente ovado; ao= estandarte ampliamente ovado).

Ejemplares examinados: COSTA RICA. Alajuela: San Mateo, mar. 1918, O. Jiménez 1218(CR); [Alajuela], Catedral [Parque central de Alajuela], jul. 1958, O. Jiménez s.n. (CR 36550, USJ 6507); San José: Acosta, San Ignacio, 1097m,
0947'55.3" N, 8409'46.0" W, 20 abr. 2019 (fr.), AguilarSandí 10 (USJ); Acosta, San Ignacio, Chirraca, 980m, $09^{\circ} 48^{\prime} 41.4^{\prime \prime} \mathrm{N}, 84^{\circ} 10^{\prime} 38.0^{\prime \prime} \mathrm{W}, 20$ abr. 2019 (fr.), AguilarSandí 11 (USJ); [Montes de Oca], [San Pedro], Universidad de Costa Rica, 5 dic. 1973, Poveda 795 (CR, JVR, USJ); Montes de Oca, Sabanilla, campus UCR, costado este del CIGRAS, entrada a UCAGRO, $1200 \mathrm{~m}, 09^{\circ} 56^{\prime} 22.4^{\prime \prime} \mathrm{N}$,

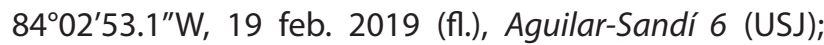
Mora, El Rodeo, Bajo Morales, 800-1000m, 09 $55^{\prime} 00^{\prime \prime} \mathrm{N}$, $84^{\circ} 16^{\prime} 00^{\prime \prime W}, 8$ jul. 1995, Q. Jiménez 1889 (CR); Mora, Zona Protectora El Rodeo, Reserva de la Universidad para la Paz, 850-900m, 0954'20" N, 84¹6'30" W, 27 may. 1997, Cascante 1324 (CR); Mora, Ciudad Colón, Hacienda el Rodeo, 900m, 0954'59.5" N, 84¹5'54.4" W, 13 abr. 2019, Aguilar-Sandí9(USJ); Santa Ana, Salitral, Chirracal, 1225m, $09^{\circ} 54^{\prime} 40.6^{\prime \prime} \mathrm{N}, 84^{\circ} 10^{\prime} 25.5^{\prime \prime} \mathrm{W}, 11$ abr. 2019, Aguilar-Sandí 7 (USJ); Santa Ana, Salitral, Chirracal, 1229m, 0954'44.1" N, 84¹0'23.6" W, 11 abr. 2019, Aguilar-Sandí 8 (USJ); Santa Ana, Salitral, Alto Raicero-Chirracal, 1220m, 09'54'22.6" $\mathrm{N}, 84^{\circ} 10^{\prime} 11.0^{\prime \prime} \mathrm{W}, 21$ abr. 2019, Aguilar-Sandí 12 (USJ).

\section{DISCUSIÓN}

Harms (1908) y Rudd (1968) reconocieron dos especies y tres variedades en Myroxylon ( $M$. peruiferum, $M$. balsamum var. balsamum, M. b. var. punctatum y M. b. var.

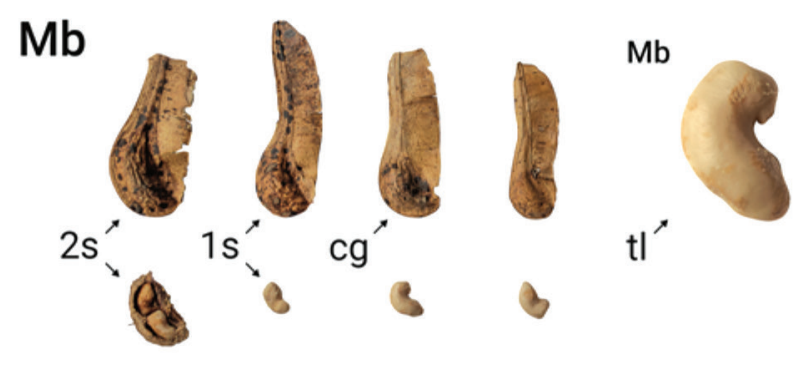

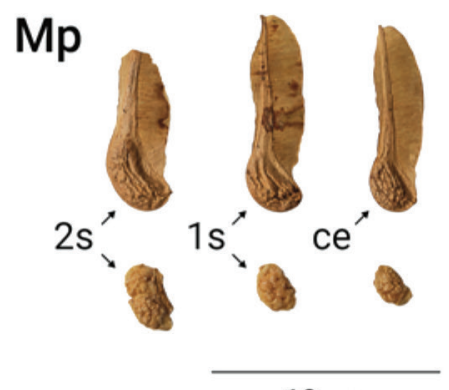

$10 \mathrm{~cm}$
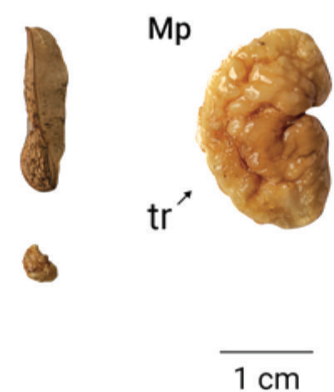

Fig. 4. Características morfológicas del fruto y la semilla para identificar y diferenciar Myroxylon peruiferum (Mp) de M. balsamum (Mb) ( $2 \mathrm{~s}=$ fruto con dos semillas; $1 \mathrm{~s}=$ fruto con una semilla; $\mathrm{cg}$ = cámara seminífera subglobosa; $c$ = cámara seminífera elíptico-aplanada; $\mathrm{tl}=$ testa lisa; $\mathrm{tr}=$ testa rugosa). 
pereirae). Sin embargo, Harms no incluyó claves dicotómicas, distribución geográfica, ni ilustraciones de estas entidades taxonómicas; por su parte, Rudd clasificó las variedades de $M$. balsamum considerando el tamaño de los frutos y las propiedades químicas de la resina, características que no son consistentes (Sartori et al., 2015).

La falta de claridad taxonómica y el uso de las características del género para identificar las especies dieron paso a que todo el material recolectado en Costa Rica se determinara como M. balsamum o M. b. var. pereirae, a pesar de la existencia de ejemplares de herbario de $M$. peruiferum recolectados en el país. Esta confusión también es patente en guías de identificación, tratamientos taxonómicos y estudios de campo (Holdridge \& Poveda, 1975; Zamora, 2010; Cascante-Marín \& Estrada-Chavarría, 2012; Jiménez, 2013).

Myroxylon balsamum y M. peruiferum presentan características morfológicas contrastantes en órganos vegetativos y reproductivos, lo que permite determinar la identidad taxonómica de ejemplares de herbario antiguos y de muestras frescas, aunque sean estériles (Sartori et al., 2015). Si bien, las características morfológicas de las flores, del fruto y de la semilla son diagnósticas, es posible diferenciar entre ambas especies considerando únicamente características vegetativas, principalmente, el tamaño de los foliolos y la forma del ápice de los mismos. En la identificación puede considerarse la procedencia de las muestras. Los árboles de M. balsamum se han recolectado en la zona sur del país (vertiente pacífica) entre 0 y $100 \mathrm{~m}$ de altitud; mientras que los árboles de M. peruiferum se recolectaron en las estribaciones montañosas al suroeste del Valle Central entre 900 y $1230 \mathrm{~m}$.

Tomando en cuenta que muchos nombres vernáculos de plantas se han recopilado en expediciones botánicas como resultado de la consulta a lugareños (León \& Poveda, 1999), entonces debe reconocerse el nombre chirraca para los árboles de $M$. peruiferum en Acosta y en Santa Ana, y no los nombres quina-quina o quina colorada de uso frecuente en países sudamericanos (Sartori et al., 2015).

En conclusión, la total ausencia de M. peruiferum en los registros de la flora costarricense se debió a la incorrecta identificación del material del género recolectado en el país y al desconocimiento de que ambas especies pueden identificarse mediante características vegetativas.

\section{AGRADECIMIENTOS}

Agradezco a Jorge Madrigal e Iván Badilla por mostrarme las chirracas que ellos conocen desde niños. A
Guillermo Quirós por darme acceso a la Hacienda El Rodeo. A Rolando Calderón por contarme de un lugar llamado Chirracal de Santa Ana. A Elmer G. García, Carlos O. Morales y Mario A. Blanco por el apoyo científico y sus sugerencias. Al personal de los herbarios CR, JVR y USJ por su colaboración. Esta investigación se deriva de mis estudios en el programa de Licenciatura en Biología con énfasis en Botánica (Escuela de Biología, Universidad de Costa Rica) y se financió con fondos propios.

\section{REFERENCIAS}

Cascante-Marín, A., \& Estrada-Chavaría, A. (2012). Las plantas vasculares de El Rodeo, Costa Rica. Brenesia, 77, 71-128.

Bolaños, R., Watson, V., \& Tosi, J. (2005). Mapa ecológico de Costa Rica (Zonas de Vida). San José, Costa Rica: Centro Científico Tropical.

MINAE. (2007). Decreto Ejecutivo No 25700. Recuperado de http://www.pgrweb.go.cr/scij/Busqueda/Normativa/ Normas/nrm_texto_completo.aspx?param 1=NRTC\&n-Valor1=1\&nValor2=29811\&nValor3=108834\&strTi$\mathrm{pM}=\mathrm{TC}$

Grayum, M. H., Hammel, B. H., \& Zamora, N. (Eds). (2016). The Cutting Edge, 23 (2). Recuperado de http://www.mobot. org/MOBOT/research/Edge/apr16/apr16lit.shtml

Harms, H. (1908). Zur Nomenclatur des Perubalsambaumes. Notizblatt des Königlichen botanischen Gartens und Museums zu Berlin, 5 (43), 85-97. https://doi. org/10.2307/3994067

Hartshorn, G. S. (1991). Plantas. En: D. H. Janzen (Ed.). Historia Natural de Costa Rica, (pp. 119-160). San José, Costa Rica: Editorial de la Universidad de Costa Rica.

Heuveldop, J., \& Espinoza, L. (1983). El componente arbóreo en Acosta y Puriscal, Costa Rica. San José, Costa Rica: Centro Agronómico Tropical de Investigación y Enseñanza.

Holdridge, L. R., \& Poveda, L. J. (1975). Árboles de Costa Rica. Volumen I. Palmas, otras monocotiledóneas arbóreas y árboles con hojas compuestas o lobuladas. San José, Costa Rica: Centro Científico Tropical.

Jiménez, Q. (1998). Árboles maderables en peligro de extinción en Costa Rica. (2da ed.). Heredia, Costa Rica: Editorial INBio.

Jiménez. Q., Rojas, F. E., Rojas V., \& Rodríguez, L. (2011). Árboles maderables de Costa Rica. Ecología y silvicultura. (2da ed.). Heredia, Costa Rica: Editorial INBio.

Jiménez, J. E. (2013). Claves dicotómicas para el reconocimiento de los árboles de El Rodeo, Valle Central de Costa Rica. Brenesia, 80, 4-35.

León, J., \& Poveda, L. J. (1999). Los nombres comunes de las plantas en Costa Rica. Heredia, Costa Rica: EFUNA.

Linnaeus filius, C. (1781). Supplementum Plantarum. Brunsvigae: Impensis Orphanotrophei. 
Limongi, R., Guiracocha, G., \& Nieto, E. (2012). Bálsamo. Myroxylon spp. Especie de uso múltiple del bosque seco del Ecuador. Estación experimental Portoviejo, Estación experimental del Litoral sur. Programa de Forestria. Boletín técnico No 152. INIAP-MAGAP-SENESCYT. Guayaquil, Ecuador: Editorial Grafiservi.

Martínez-Rivera, J. C., Blanco, J., Colorado, R., Guerra, O., \& Antonio, H. (2015). El impacto cultural del árbol de bálsamo en Chiltiupán, La Libertad, El Salvador. Revista Entorno, 58, 25-33. https://doi.org/10.5377/entorno. v0i58.6238

Meza, T., \& Bonilla, A. (1990). Áreas naturales protegidas de Costa Rica. Cartago, Costa Rica: Editorial Tecnológica de Costa Rica.
Rudd, V. E. (1968). Leguminosae of Mexico-Faboideae. I. Sophoreae and Podalyrieae. Rhodora, 70, 493-532.

Sartori, A. L. B, Lewis, G. P., Mansano, V.F., \&Tozzi, A. M. G. A. (2015). A revision of the genus Myroxylon (Leguminoseae: Papilionoideae). Kew Bulletin 70, 48 (1-12). DOI: 10.1007/ S12225-015-9604-7

Zamora, N. (2010). Fabaceae. En: B. E. Hammel, M. H. Grayum, C. Herrera \& N. Zamora (Eds.). Manual de Plantas de Costa Rica. Vol. V. Dicotiledóneas (Clusiaceae-Gunneraceae). Monographs in Systematic Botany from the Missouri Botanical Garden, 119, 395-775. 